\title{
The Mediation Effect of Information Presentation Style on the Relationship between Banner Advertisements and Advertising Effectiveness
}

\author{
Hsin Kuang $\mathrm{Chi}^{1}$, Huery Ren $\mathrm{Yeh}^{2} \&$ Cherng-Ying Chiou ${ }^{3}$ \\ ${ }^{1}$ Department of Business Administration, Nan Hua University, Taiwan \\ ${ }^{2}$ Department of Marketing Management, Shih Chien University, Kaoshiung, Taiwan \\ ${ }^{3}$ Department of International Trade \& Logistics, Overseas Chinese University, Taiwan \\ Correspondence: Cherngying Chiou, Department of International Trade \& Logistics, Overseas Chinese \\ University, Taiwan. Tel: 886-4-2701-6855. E-mail: a00000965@gmail.com
}

Received: April 9, 2012 Accepted: May 22, 2012 Online Published: July 16, 2012

doi:10.5539/ijbm.v7n14p46 URL: http://dx.doi.org/10.5539/ijbm.v7n14p46

\begin{abstract}
Due to the rapid development of information technology in recent years, internet has become a main avenue for people to receive daily information. It is also an important tool for advertisers to publish their advertisements in the internet surroundings. However, the information presentation style on the internet will probably influence the viewer's intention to click banner advertisements and further influence the advertisement effectiveness. The study takes the banner advertisement as the independent variable, the advertising effectiveness as the dependent variable, and the information presentation style as the mediation variable to separately explore the influence of the banner advertisement on the advertising effectiveness under two different styles (obtrusive and contextual) on a website. The study uses the internet browsers (university computer lab students, $3 \mathrm{C}$ hypermarket customers, and office staffs) who get accustomed to surfing a website as survey objects. 550 questionnaires were sent out and 422 copies were valid. The findings of the research result are as follows: (1) both the banner advertisement and obtrusive information presentation style have a positive influence on the advertising effectiveness; (2) the contextual information presentation style does not have a mediation effect on the banner advertisement and advertising effectiveness but the obtrusive information presentation style does.
\end{abstract}

Keywords: banner advertisement, information presentation style, advertising effectiveness, mediation effect

\section{Introduction}

In past decades, the rapid progress in the information technology made the internet become one of people's main avenues to receive, browse and search information. The marketing managers lay more emphases on the internet surroundings to promote the product and deliver the message (Ha, 2003; Leong \& Huang, 1998; Macias, 2003; Sicilia, Ruiz, \& Munuera, 2005; Strauss \& Frost, 2001). The banner advertisement is the most common format in the internet advertisements, and it is visible everywhere when people surf a homepage. Comparing with the traditional advertisement and banner advertisement not only helps the advertiser in delivering the advertisement information but also provides an interactive channel between the internet user and the advertisement (Zeff \& Aronson, 1999). By connecting another hyperlink homepage through clicking a banner advertisement, the advertiser can promote more complete advertisement information and increase the advertising effectiveness. Users can also quickly acquire the advertisement information they needed. This kind of interactive advertisements become a unique marketing medium in the internet ads and influence users' evaluation on an advertisement and advertisement content (Raney, Arpan, Pashupati, \& Brill, 2003; Ko, Cho, \& Roberts, 2005; Sundar \& Kim, 2005). There are many factors able to influence banner advertisement effectiveness. The most important factor is the information presentation style of an advertisement that affects whether browsers will click a banner advertisement or not (Kuo \& Chang, 2004). In addition, whether users can identify an advertisement (Ariely, 2000; Sundar \& Kim, 2005) and product involvement are probable other critical reasons influencing banner advertising effectiveness (Lin \& Lin, 2005). Accordingly, advertisers should pay attention to selecting a suitable information presentation style and make a good banner advertisement to satisfy the user's information requirement since the information presentation style and banner advertisement are highly related to the 
advertising effectiveness.

The study attempts to understand which information presentation style (obtrusive or contextual) can produce a good result between the banner advertisement and advertising effectiveness. Thus, the study aims to (1) explore the influence of the banner advertisement and the information presentation style on the advertising effectiveness, (2) explore the influence of the banner advertisement on the advertising effectiveness under two different information presentation styles, and (3) verify whether the information presentation style is the mediation variable between the banner advertisement and advertising effectiveness.

\section{Literature Review}

\subsection{Banner Advertisement}

The advertisers spread out the advertisement information via all kinds of media to persuade the audience to buy their products or services. These media include television broadcast, press and internet advertisement. The internet advertisement, in particular, has deeply emphasized in recent years because of time saving and the progress in the internet technology. Zeff and Aronson (1999) and Baker and Gronne (1996) consider that the internet advertisement influences a browser's behavior by delivering messages through a website, and it is different from other advertisement media because of its interaction effect between a browser and the internet advertisement. Thus, a browser can gain more information through clicking an advertisement and further buy a product online. As a result, the internet advertisement gradually transform from the role of serving as one-way message delivery to an interactive medium (Baker \& Gronne, 1996).

A banner advertisement is a popular homepage advertisement, and it combines words and pictures with the obtrusive or contextual presentation style in a webpage to attract a user to click and interact with an advertisement and buy a product online. The features of the banner advertisement are two-way interactive communication that a browser can possess more autonomy than ever to determine whether further read the advertisement or not and find information quickly and effectively (Weng, 2003; Zeff \& Aronson, 1999). Placing a banner advertisement on a well-known website can have more internet users to browse a brand or a product and increase its awareness and economic benefit (Briggs \& Stipp, 1999). Moreover, the manufacturing cost is cheaper and manufacturing time for a banner advertisement is shorter so that the advertiser can quickly replace the advertisement with a bad response.

\subsection{Information Presentation Style}

Goldfarb and Tucker (2011) divide online information presentation style into contextual style and obtrusive style. Contextual style is a banner advertisement which only displays static information on a website while obtrusive style is a banner advertisement which applies pop-up, pop-under, in-stream, takeover, non-user-initiated video and audio, interstitial, non-user-initiated background music, full-page banner, interactive and floating ads on a website (Goldfarb \& Tucker, 2011).

In the visual stimulus, the obtrusive object or image has a greater stimulative effect than a contextual object (Wang \& Chen, 2004; Ting, 1999; Vaughan, 1993) because it can attract more browsers' attention to the information presenting on the screen. However, contextual contents receive less response and confine the advertising effectiveness, although it is easy to make and accept by all websites (Guo, 2001).

Thus, an obtrusive banner advertisement sends more fruitful information and holds a continuous action so that it can attract browsers to make a click and increase click-through rate. Nevertheless, when an obtrusive banner advertisement applies too much annoying technology, it can increase objection and dissatisfaction and produces an irritable, angry and negative attitude to a browser because of negative advertisement feelings (Ducoffe, 1996). Therefore, it is a very important issue for advertisers to select an appropriate information presentation style to persuade a browser to buy a brand or a product directly online.

\subsection{Advertising Effectiveness}

The main functions of a banner advertisement are to provide a browser with product information and to arouse his or her interest and purchase intention. If an advertisement provides more detailed product information, the advertising effectiveness will be higher. The advertising effectiveness is a kind of communication for advertisers to convey advertisement information to consumers (Ducoffe, 1996), and how to measure advertising effectiveness has been a research interest for advertisement scholars and advertisement proprietors. Among a lot of measurement methods, click-through rate is the most widely used measure to test the advertising effectiveness of a banner advertisement (Lambercht \& Tucker, 2011). Compared with other measuring method, the correlation of the click-through rate and banner advertisement is very high, and an advertiser also hopes that users can click to the advertiser's website after surfing a banner advertisement (IAB, 1997; Hoffman \& Novak, 1996; Briggs \& 
Stipp, 1999). Hence, many commercial website advertisers often take the click-through rate as a criterion for the base of the advertisement expenditure.

Lavidge (1961) divides the advertising effectiveness into hit effect and communication effect. The former takes whether the hit amount increases as a measuring criterion to measure whether consumers have the will and tendency to click an advertisement; the latter measures the extent of information are paid attention to and the attitude and behavior change of consumers. The research of Cho (1999) finds that an obtrusive banner advertisement has a higher click will. Hence, with a view to understanding the behavior produced by the advertisement after consumers are stimulated, the study uses the intention to click advertisement and attraction effect to measure the advertising effectiveness. Every advertiser hopes that the banner advertisement will be clicked but the click-through rate of the banner advertisement is generally less than $1 \%$ (Kuo \& Chang, 2004). If advertisers want to increase internet users to click an advertisement, they have to maintain the creativity consecutively and present a new guise on their advertisements to attract the user's eye. Besides, planning a more accurate target and selecting objects who surf a certain website can increase the click-through rate from the objective browsers.

\subsection{The influence among Banner Advertisement, Information Presentation Style and Advertising Effectiveness}

Huang, $\mathrm{Hu}$ and $\mathrm{Wu}$ (2008) find that the animated presentation is the best for the advertising effect, while the contextual presentation gets worse effect. The more fruitful information presentation is; the higher the user's attention and advertisement effect can be raised (Hoffman and Novak, 1996; Wang \& Chen, 2004). The more obvious of an advertising effectiveness is, the more advertisement attitude and attraction are (Cho, 1999; Huang, $\mathrm{Hu}, \& \mathrm{Wu}, 2008)$. The reasons why a banner advertisement succeeds are its information presentation style to make the browsers click the advertisement and understand the advertisement content and further facilitate their purchase intention (Briggs \& Stipp, 1999; Weng, 2003). Pursuant the foresaid literature, the information presentation style is deduced that the more diversified the medium is, the better the advertising effect is, and different information presentation styles have a different influence on the banner advertisement effect.

\section{Research Methodology}

\subsection{Research Framework}

The research framework of the study is shown on Figure 1. The study takes the banner advertisement as the independent variable, the advertising effectiveness as the dependent variable, and the information presentation style as the mediation variable to explore whether the advertising effectiveness has significant effect.

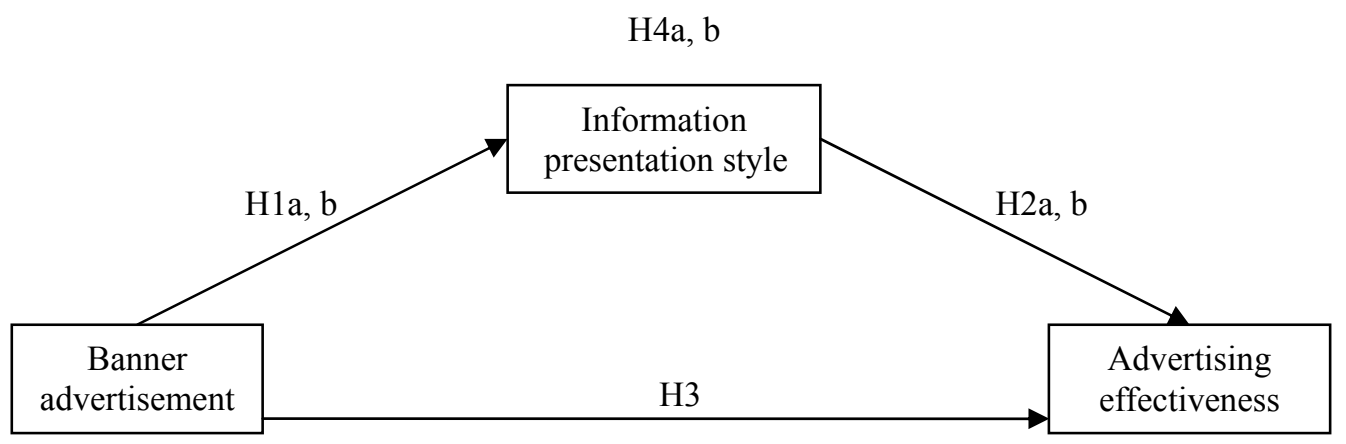

Figure 1. Research framework

\subsection{Research Hypotheses}

H1a: The banner advertisement has a significantly positive influence on the obtrusive information presentation style.

H1b: The banner advertisement has a significantly positive influence on the contextual information presentation style.

H2a: The obtrusive information presentation style has a significantly positive influence on the advertising effectiveness. 
$\mathrm{H} 2 \mathrm{~b}$ : The contextual information presentation style has a significantly positive influence on the advertising effectiveness.

H3: The banner advertisement has a significantly positive influence on the advertising effectiveness.

H4a: The obtrusive information presentation style is a mediation variable which exists between the banner advertisement and the advertising effectiveness.

H4b: The contextual information presentation style is a mediation variable which exists between the banner advertisement and the advertising effectiveness.

\subsection{Research Scale and Sample}

The research tool of the study is the questionnaire. The questions in the questionnaire are measured by the 7-point Likert scale, "1-very agree/7-very disagree". The banner advertisement is defined as a popular homepage advertisement that combines words and pictures into the obtrusive or contextual presentation style on a webpage to attract a user to click and interact with an advertisement. There are five questions in the banner advertisement scale, and the Cronbach' $\alpha$ is 0.783 . The information presentation style divides into the obtrusive style and contextual style, which combines dynamic or static words and pictures to present advertisement messages on the webpage to the viewers. Obtrusive presentation style includes animations, images and voices while contextual presentation style contains static words and pictures presenting in the motion frame. Information presentation style has eleven questions originally. One question was deleted because its loading factor was 0.232 . The Cronbach' $\alpha$ is 0.723 . The advertisement effectiveness denotes a browser's click intention after viewing a banner advertisement. The advertisement effectiveness scale has eight questions, and the Cronbach' $\alpha$ is 0.919 .

Thus, the Cronbach's $\alpha$ value of every variable is greater than 0.7 in this research. Furthermore, the study takes the internet users (university computer lab students, 3C hypermarket customers and office staffs) who get accustomed to surfing the website as survey objects and adopts convenience sampling to collect data. The study sent out 550 questionnaires and 453 questionnaires were returned. The valid questionnaires are 422 after deducting 31 invalid questionnaires. The valid response rate is $77 \%$.

\section{Research Results}

\subsection{Sample Feature}

The sample features are interpreted as follows: total 422 samples were collected in Chiayi County, Taiwan including 190 university computer lab students (45\%), 148 3C hypermarket customers (35\%), and 84 company office staffs (20\%). 214 (50.7\%) are males and 208 (49.3\%) are females. Aged 21-30 accounts for 241(57.1\%), and aged 31-40 accounts for 104 (24.6\%). 99 (23.5\%) are married and 323 (76.5\%) are single. 230 (50.2\%) own bachelor degree, and $112(26.5 \%)$ are above master degree. 133 (31.5\%) earn monthly salary NT $\$ 30,001-N T \$ 40,000$, and $97(23.0 \%)$ earn less than NT\$20,000. The conditions for users surfing a website weekly are $34(8.1 \%)$ less than 10 hours, 70 (16.6\%) 11-20 hours, 89 (21.1\%) 21-30 hours, $53(12.6 \%)$ 31-40 hours, $63(14.9 \%) 41-50$ hours and $113(26.8 \%)$ over 51 hours.

\subsection{Correlation Analysis}

In the Pearson correlation analysis, the results show that the banner advertisement and advertising effectiveness have a significantly positive correlation $(\mathrm{r}=0.351, \mathrm{P}<0.01)$, and the banner advertisement and the obtrusive information presentation style have a significantly positive correlation $(\mathrm{r}=0.258, \mathrm{P}<0.01)$. In addition, the banner advertisement and the obtrusive information presentation style do not have a significantly positive correlation $(\mathrm{r}=-0.041)$, but the obtrusive information presentation style and advertising effectiveness have a significantly positive correlation $(r=0.569, \mathrm{P}<0.05)$, and the contextual information presentation style $(\mathrm{r}=0.017)$ does not have a significantly positive correlation with the advertising effectiveness.

\subsection{Verification of Mediation Effect}

The research verifies whether the banner advertisement influences the advertising effectiveness via the mediation of the information presentation style. As shown in Table 1, Model 1 indicates that the banner advertisement has a significantly positive influence on the obtrusive information presentation style $(\beta=0.258, p=0.000)$, and the banner advertisement does not have a significantly positive influence on the contextual information presentation style $(\beta=-0.041, \mathrm{p}=0.400)$; therefore, H1a is supported but $\mathrm{H} 1 \mathrm{~b}$ is rejected. Pursuant to the verification of the mediation effect suggested by Baron and Kenny (1986), the significant standard regression coefficient has to exist between the independent variable and mediation variable. In Model 2, the banner advertisement has a significant positive influence on the advertising effectiveness $(\beta=0.351, \mathrm{p}=0.000<0.05)$, so $\mathrm{H} 3$ is supported. The study further takes the banner advertisement as an independent variable to regress with and the advertising 
effectiveness, the dependent variable. The result shows that the obtrusive information presentation style has a significantly positive influence on the advertising effectiveness ( $\beta=0.569, \mathrm{p}=0.000<0.05$ ), so H2a is supported. However, $\mathrm{H} 2 \mathrm{~b}$ is not supported $(\beta=0.017, \mathrm{p}=0.727$ ).

In addition, in the model 3 , when the mediating variable, the obtrusive information presentation style, is controlled, it shows that the advertising effectiveness and obtrusive information presentation style are significantly affected to the banner advertisement $(\mathrm{p}=0.000<0.05)$, and the regression coefficient of the advertising effectiveness reduces from 0.351 to 0.219 . Pursuant to the verification of the mediation effect suggested by Baron and Kenny (1986), the obtrusive information presentation style has part of the mediation effect in the influence of the banner advertisement on the advertising effectiveness, and the partial mediation effect is 0.132 , so H4a is supported. However, although banner advertisement is significantly affected to the advertising effectiveness $(\beta=0.353, \mathrm{p}=0.000<0.05)$, contextual information presentation style is not significantly $(\beta=0.032, p=0.491)$, so $\mathrm{H} 4 \mathrm{~b}$ is not supported.

Table 1. Regression analysis of banner advertisement effectiveness

\begin{tabular}{|c|c|c|c|c|c|c|c|}
\hline \multirow{3}{*}{ Variable } & \multicolumn{2}{|c|}{ Model 1} & \multicolumn{3}{|c|}{ Model 2} & \multicolumn{2}{|c|}{ Model 3} \\
\hline & \multicolumn{2}{|c|}{ Style } & \multirow{2}{*}{\multicolumn{3}{|c|}{ Advertising effectiveness }} & \multirow{2}{*}{\multicolumn{2}{|c|}{$\begin{array}{c}\text { Advertising } \\
\text { effectiveness }\end{array}$}} \\
\hline & Obtrusive & Contextual & & & & & \\
\hline \multirow{2}{*}{$\begin{array}{c}\text { Banner } \\
\text { advertisement }\end{array}$} & $0.258^{*}$ & -0.041 & $0.351^{*}$ & & & $0.219^{*}$ & $0.353 *$ \\
\hline & $(.000)$ & $(.400)$ & $(.000)$ & -- & -- & $(.000)$ & $(.000)$ \\
\hline Obtrusive & -- & -- & -- & $\begin{array}{l}0.569 * \\
(.000)\end{array}$ & -- & $\begin{array}{l}0.513^{*} \\
(.000)\end{array}$ & -- \\
\hline Contextual & -- & -- & -- & -- & $\begin{array}{l}0.017 \\
(.727)\end{array}$ & -- & $\begin{array}{l}0.032 \\
(.491)\end{array}$ \\
\hline Adj. $R^{2}$ & 0.064 & -0.001 & 0.121 & 0.322 & -0.002 & 0.366 & 0.120 \\
\hline$F$-value & 29.867 & 0.711 & 59.182 & 201.267 & 0.122 & 122.449 & 29.792 \\
\hline
\end{tabular}

\section{Conclusion}

As shown in Table 1, in this research, banner advertisement influences advertising effectiveness via mediation effect of the information presentation style. The study verifies that both the banner advertisement and obtrusive information presentation style has a positive influence on the advertising effectiveness, but the contextual information presentation style does not have a positive influence on the advertising effectiveness. Accordingly, delivering the advertisement information on the homepage via the animated word and picture, image and voice with fixed action frame to the user, the advertising effectiveness will subsequently rise. In using the contextual information presentation style, the advertising effectiveness is not significantly influenced. It is supported that the mediation effect exists in the obtrusive information presentation style, and the contextual information presentation style is not supported. The results show that the obtrusive information presentation style has an indirect prediction effect and additional function on the advertising effectiveness on the internet users because it usually includes animation, image and voice, etc., and all of picture presentations possess the animated action can attract browsers; for example, a pop-up word, a changing image, a picture spreading all of a sudden, and voice special efficacy collocation, all of these can attract the user's eye to click the presentation in surfing. On the other hand, the contextual information presentation style only shows words or pictures with a stationary action. Such style is easily ignored or cannot further raise the click interest, so the contextual information presentation style cannot produce an obvious benefit on the internet user.

In the meantime, the study suggests that advertisers should adopt an obtrusive banner advertisement in that a browser is easily attracted by this kind of information presentation style and further achieve the advertising effectiveness. Additionally, the study finds that over half the survey respondents do not especially dislike or have a negative perception on the display banner advertisement probably because the internet users get accustomed to all kinds of forceful internet advertisements (e.g. a pop-up advertisement) and maintain a positive perception. 
Therefore, an advertiser can stress the use of the obtrusive banner advertisement to transmit the advertisement information to increase the advertisement effect. Moreover, the study recommends that for a future research a researcher can add product preference or product involvement into investigation to further understand browsers' actual clicking effect since the present study only examines browsers' intention to click a banner advertisement on a website.

\section{References}

Ariely, D. (2000). Controlling the information flow: Effects on consumers' decision making and preferences. Journal of Consumer Research, 27(2), 233-248. http://dx.doi.org/10.1086/314322

Barker, C., \& Gronnes, P. (1996). Advertising on the World Wide Web. Unpublished master's thesis, Copenhagen Business School, Danmark. Retrieved from http://www.pg.dk/pg/advertising

Baron, R. M., \& Kenny, D. A. (1986). The moderator-mediator variable distinction in social psychological research: Conceptual, strategic, and statistical consideration. Personality and Social Psychology Review, 51(6), 1173-1182. http://dx.doi.org/10.1037/0022-3514.51.6.1173

Briggs, R., \& Stipp, H. (1999). How internet advertising works. Retrieved from http://www.mbinteractive.com/resources/whitepapers.html.

Cho, C. (1999). How advertising works on the World Wide Web: Modified elaboration likelihood model. Journal of current issues and research in advertising, 21(1), 33-49.

Ducoffe, R. H. (1996). Advertising value and advertising the web. Journal of advertising of research, 36(5), 21-35.

Goldfarb, A., \& Tucker, C. (2011). Online display advertising: Targeting and obtrusiveness. Marketing Science, 30(3), 389-404. http://dx.doi.org/10.1287/mksc.1100.0583

Guo, C. (2001). The effects of cognitive style and involvement on banner advertising. Unpublished master's thesis, National Chung Cheng University, Taiwan.

Ha, L. (2003). Crossing offline and online media: A comparison of online advertising on TV web sites and online portals. Journal of Interactive Marketing. Retrieved from http://www.jiad.org/vol3/no2/ha/ha.pdf.

Hoffman, D. L., \& Novak, T. P. (1996). Marketing in hypermedia computer-mediated environments: Conceptual foundations. Journal of Marketing, 60(3), 50-69. http://dx.doi.org/10.2307/1251841

Huang, L. Y., Hu, A. N., \& Wu, K. L. (2008). The effects of multimedia message service on mobile advertisement. Marketing Review, 5(4), 481-513.

IAB. (1997). Metrics and methodology by the media measurement task force. Retrieved from http://www.iab.net/advertise/content/mmtf3.html\#0

Ko, H., Cho, C. H., \& Roberts, M. S. (2005). Internet uses and gratifications: A structural equation model of interactive advertising. Journal of Advertising, 34(2), 57-70.

Kuo, C., \& Chang, C. C. (2004). What would you do if you were tricked? Examining the effects trick banners. Management Review, 23(3), 53-70.

Lambrecht, A., \& Tucker, C. (2011). When does retargeting work? Timing information specificity. Retrieved from http://ssrn.com/abstract=1795105

Lavidge, R. J. (1961). A model for predictive measurements of advertising effectiveness. Journal of Marketing, 25(6), 59-62. http://dx.doi.org/10.2307/1248516

Leong, E. K. F., \& Huang, X. (1998). Comparing the effectiveness of the web site with traditional media. Journal of Advertising Research, 38(5), 44-51.

Lin, L. Y., \& Lin, Y. M. (2005). The influence of advertising comparative strategy and advertising appeals on purchasing intention: The moderate effect of product involvement. Journal of Business Administration, 65, $1-29$.

Macias, W. (2003). A preliminary structural equation model of comprehension and persuasion of interactive advertising brand web sites. Journal of Interactive Marketing, 3(2). Retrieved from http://www.jiad.org

Raney, A. A., Arpan, L. M., Pashupati, K., \& Brill, D. A. (2003). At the movies, on the web: An investigation of the effects of entertaining and interactive web content on site and brand evaluations. Journal of Interactive 
Marketing, 17(4), 38-53. http://dx.doi.org/10.1002/dir.10064

Sicilia, M., Ruiz, S., \& Munuera, J. L. (2005). Effects of interactivity in a web site: The moderating effect of need for cognition. Journal of Advertising, 34(3), 31-45.

Strauss, J., \& Frost, R. (2001). E-Marketing. New Jersey: Prentice Hall.

Sundar, S. S., \& Kim, J. (2005). Interactivity and persuasion: Influencing attitudes with information and involvement. Journal of Interactive Marketing, 5(2), 6-29.

Ting, Y. H. (1999). A Study of Advertising Effects of Different Media Properties, Advertising Appeals, and Product Involvement-A Comparison between Print Media and the Internet. Unpublished master's thesis, National Sun Yat-sen University, Taiwan.

Vaughan. (1993). Multimedia: Make it work. New York: McGraw-Hill.

Wang, N. T., \& Chen, S. P. (2004). A consumer attitude study of web advertisement expressions style-visual and acoustic. Journal of National Taiwan College of Arts, 75, 71-86.

Weng, W. J. (2003). The effects of designed exploration methods of internet advertisements on advertising values, advertising effects: Discuss of the moderate effects of involvement of products and advertisements. Unpublished master's thesis, Shih Chien University, Taiwan.

Zeff. R., \& Aronson, B. (1999). Advertising on the internet (2nd ed.). New York, John Wiley \& Sons, Inc. 